\title{
Reactor Noise Analysis for a Graphite-moderated and -reflected core in KUCA
}

\author{
Atsushi SAKON ${ }^{1 *}$, Kunihiro NAKAJIMA ${ }^{2}$, Kazuki TAKAHASHI ${ }^{2}$, Shin-ya HOHARA ${ }^{1}$, \\ Tadafumi SANO $^{3}$, Yuji FUKAYA ${ }^{4}$, and Kengo HASHIMOTO ${ }^{1}$
}

\author{
${ }^{1}$ Atomic Energy Research Institute, Kindai University \\ 3-4-1 Kowakae, Higashi-Osaka city, Osaka, Japan \\ ${ }^{2}$ Graduate School of Science and Engineering, Kindai University \\ 3-4-1 Kowakae, Higashi-Osaka city, Osaka, Japan \\ ${ }^{3}$ Institute for Integrated Radiation and Nuclear Science, Kyoto University \\ 2nd Asashiro-nishi, Kumatori-cho, Sennan gun, Osaka, Japan \\ ${ }^{4}$ Japan Atomic Energy Institute \\ 4002 Narita-cho Shinbori, Oarai-cho, Higashi-Ibaraki gun, Ibaraki, Japan
}

Sakon@kindai.ac.jp, kunihiro_nakajima@nk-mail.com, takahashi.kazuki0524@gmail.com, hohara@kindai.ac.jp, t-sano@kindai.ac.jp, fukaya.yuji@jaea.go.jp, kengoh@pp.iij4u.or.jp

\begin{abstract}
In graphite-reflected thermal reactors, even a detector placed far from fuel region may detect a certain degree of the correlation amplitude. This is because mean free path of neutrons in graphite is longer than that in water or polyethylene. The objective of this study is experimentally to confirm a high flexibility of neutron detector placement in graphite reflector for reactor noise analysis. The present reactor noise analysis was carried out in a graphite-moderated and -reflected thermal core in Kyoto University Critical Assembly (KUCA). $\mathrm{BF}_{3}$ proportional neutron counters (1" dia.) were placed in graphite reflector region, where the counters were separated by about $35 \mathrm{~cm}$ and $30 \mathrm{~cm}$-thick graphite from the core, respectively. At a critical state and subcritical states, time-sequence signal data from these counters were acquired and analyzed by a fast Fourier transform (FFT) analyzer, to obtain power spectral density in frequency domain. The auto-power spectral density obtained from the counters far from the core contained a significant degree of correlated component. A least-squares fit of a familiar formula to the auto-power spectral density data was made to determine the prompt-neutron decay constant. The decay constant was $63.3 \pm 14.5[1 / \mathrm{s}]$ in critical state. The decay constant determined from the cross-power spectral density and coherence function data between the two counters also had a consistent value. It is confirmed that reactor noise analysis is possible using a detector placed at about $35 \mathrm{~cm}$ far from the core, as we expected.
\end{abstract}

KEYWORDS: Graphite core, Reactor noise, Power Spectrum method, Prompt-Neutron Decay Constant 


\section{INTRODUCTION}

Research and development of High Temperature Gas-cooled Reactor (HTGR) has been carried out in High Temperature Engineering Test Reactor (HTTR) of The Japan Atomic Energy Agency (JAEA) [1-5]. Graphite core reactor is expected as an option for Small Modular Reactors (SMR) or Innovative nuclear reactor. There are few recent researches of reactor physics experiments in graphite-reflected reactors, and reactor noise analysis is particularly minor. In light-water- or polyethylene- reflected thermal reactors, neutron detector for a reactor noise analysis must be placed closely to fuel region to observe a neutron correlation amplitude of significance. For example, a detector separated by a $30 \mathrm{~cm}$-thick water reflector from fuel region must have no neutron correlation information. The neutron mean free path in graphite is longer than that of light water and polyethylene. In graphite-reflected reactors, detectors away from the fuel region may be able to detect the correlation component of reactor noise. The objectives this study is to examine experimentally advantage of graphite-reflected reactor for noise analysis, and adverse effect from neutron source. To achieve these goals, a series of power spectral analysis experiments were performed in a graphite-reflected thermal core of the Kyoto University Critical Assembly (KUCA). This experiment was carried out in a critical state and in subcritical states driven by Am-Be neutron source. In this paper, the experimental configuration, measurement system and analysis method are described in Section 2. Results of the Power spectrum analysis are described in Section 3. Conclusion of this study is described in Section 4.

\section{EXPERIMENT}

The core configuration and neutron detector placement are shown in Figure 1. The core was composed of 25 low-enrichment mimic fuel assemblies (7/4"G2/8"p8EUNU), 18 regular driver fuel assemblies (3/8"p38EU), 3 partial driver fuel assemblies (3/8"p18EU and 3/8"p14EU) and many graphite reflector assemblies surrounding the fuel assemblies. The low-enrichment mimic fuel assembly was composed of 8 unit cells. The unit cell of this fuel assembly was composed of one (1/16" thick) enriched uranium plate, one (1-mm thick) natural uranium plate, four graphite plate (three $1 / 2$ " thick plates and one $1 / 4$ " thick plate) and two (1/8" thick) polyethylene plates. The regular driver fuel assembly was composed of 38 unit cells, and partial driver fuel assembly was composed of 18 or 14 unit cells. The unit cell of driver fuel assembles was composed of one (1/16" thick) enriched uranium plate and three (1/8" thick) polyethylene plates. The partial driver fuel assembly was employed to adjust the criticality of the core. Extended graphite reflector area for placement of detectors was constructed laterally from the core. In this area, four $\mathrm{BF}_{3}$ detectors (1" dia.) were placed at intervals of 3" from the vicinity of the fuel assemblies (K-1, K-4, $\mathrm{K}-7$ and $\mathrm{K}-10$ in Figure 1). In addition to these detectors, two $\mathrm{BF}_{3}$ detectors (1" dia.) were placed in different directions (B-15 and R-15 in Figure 1).

KUCA has six control rods, where three are assigned as safety rods for reactor SCRAM and are referred to as S4, S5, and S6, respectively. Others are rods for adjustment of reactivity and are referred to as $\mathrm{C} 1$, $\mathrm{C} 2$, and $\mathrm{C} 3$, respectively. The subcritical reactivity for the present experiment was adjusted by changing the axial positions of these safety and control rods. The rod patterns employed in the experiment are shown in Table I. The reference reactivity, included in this table, was evaluated from the reactivity worth of each rod, which was predetermined by the positive period method and the rod drop one [6]. The subcritical core was driven by an Am-Be neutron source (I-25 in Figure 1).

In the power spectrum analysis, the time sequence data of the detectors are analyzed by a Fast Fourier Transformer (FFT-analyzer) to obtain the auto-power spectral density and the cross-power spectral density between any two detectors. The present neutron instrumentation system is shown in Figure 2. The rate meter converts time-sequence pulse signals into analog voltage output proportional to the instantaneous count rate of the input pulses. The time constant about $3.7 \mathrm{~ms}$ of the meter is so short that a large portion of the reactor noise passes through the filtration. 
In this measurement, detectors $\mathrm{B} 2$ and $\mathrm{B} 5$ were used for patterns $\mathrm{S}, \mathrm{A}$, and $\mathrm{B}$ due to the limitation of the number of measurement circuits. The pattern $\mathrm{C}$ is low counting of the detector $\mathrm{B} 2$ and $\mathrm{B} 5$, it could not be subject to the power spectrum analysis. Therefore, using the detector B4 and B6 in the pattern C.

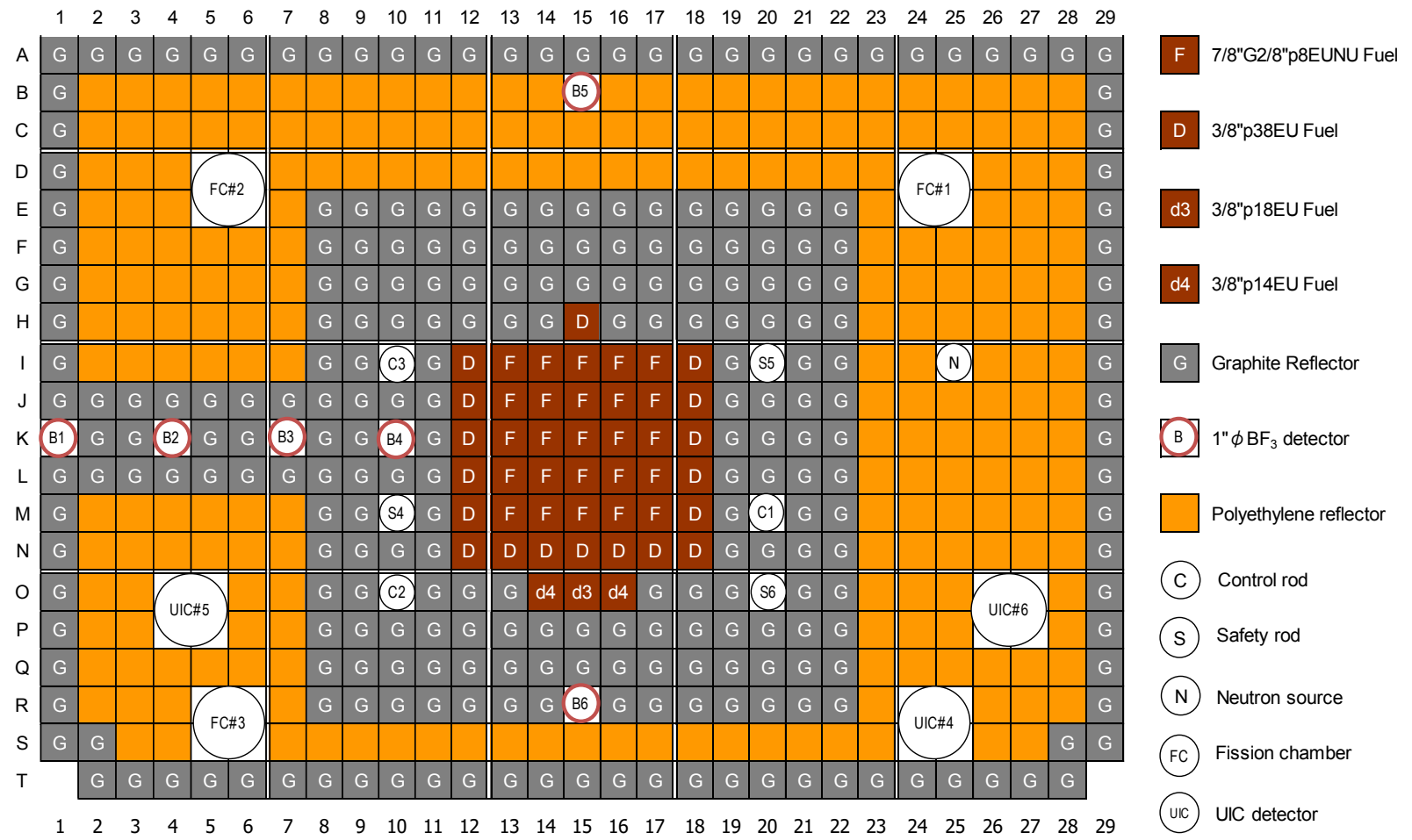

Figure 1 Top view of core configuration and detector location.

Table I. Rod Patterns of employed in the present experiment.

\begin{tabular}{|c|c|c|c|c|c|c|c|}
\hline \multirow{2}{*}{$\begin{array}{c}\text { Rod } \\
\text { Pattern }\end{array}$} & \multicolumn{6}{|c|}{ Control and Safety Rod Position } & \multirow{2}{*}{$\begin{array}{c}\text { Reactivity* } \\
{[\% \Delta \mathrm{k} / \mathrm{k}]}\end{array}$} \\
\cline { 2 - 7 } & C1 & C2 & C3 & S4 & S5 & S6 & (critical) \\
\hline S & U.L. & U.L. & $675.48 \mathrm{~mm}$ & U.L. & U.L. & U.L. & 0.738 \\
\hline A & L.L. & U.L. & U.L. & U.L. & U.L. & U.L. & 0.71 \\
\hline B & L.L. & L.L. & L.L. & U.L. & U.L. & U.L. & 1.871 \\
\hline C & L.L. & L.L. & L.L. & L.L. & L.L. & L.L. & 3.942 \\
\hline
\end{tabular}

$*$ Evaluated from reactivity worth of the control rod.

L.L., lower limit; U.L., upper limit.

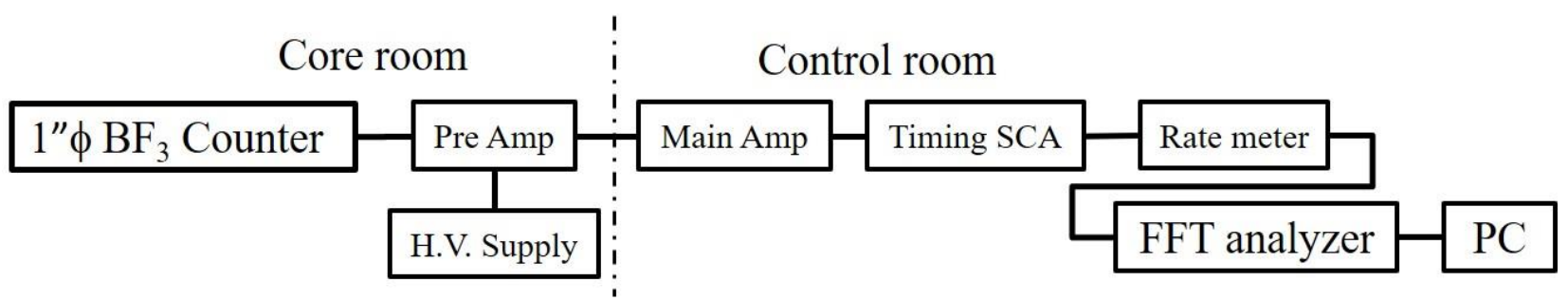

Figure 2. Measurement system of employed in the present experiment. 


\section{RESULTS AND DISCUSSION}

Figure 3 shows measured auto-power spectral density from neutron detector B2 and B5 in rod pattern $\mathrm{S}$ (critical state). The power spectral density is real, and accordingly, only the magnitude of the spectral density is presented in this paper. The prompt-neutron decay constants $\alpha_{0}$ were obtained by performing a least-squares fit of a familiar Equation [7] to the auto-power spectral densities from each detector. The decay constants were consistent with $63.1[1 / \mathrm{s}]$ calculated by the continuous-energy Monte Carlo code MVP version 3 [8] with evaluated nuclear data library of JENDL-4.0 [9] within statistical uncertainty. It is confirmed that reactor noise analysis is possible using a detector placed at about $35 \mathrm{~cm}$ far from the core, as we expected. The distance between the detector and the fuel region is about the same as $35 \mathrm{~cm}$ (B2) and $30 \mathrm{~cm}$ (B5). However, the spectral intensity of the auto-power spectral density of detector B5 is attenuated by an order of magnitude or more compared to detector B2. It was confirmed that the correlation component of reactor noise attenuated greatly through the polyethylene region.

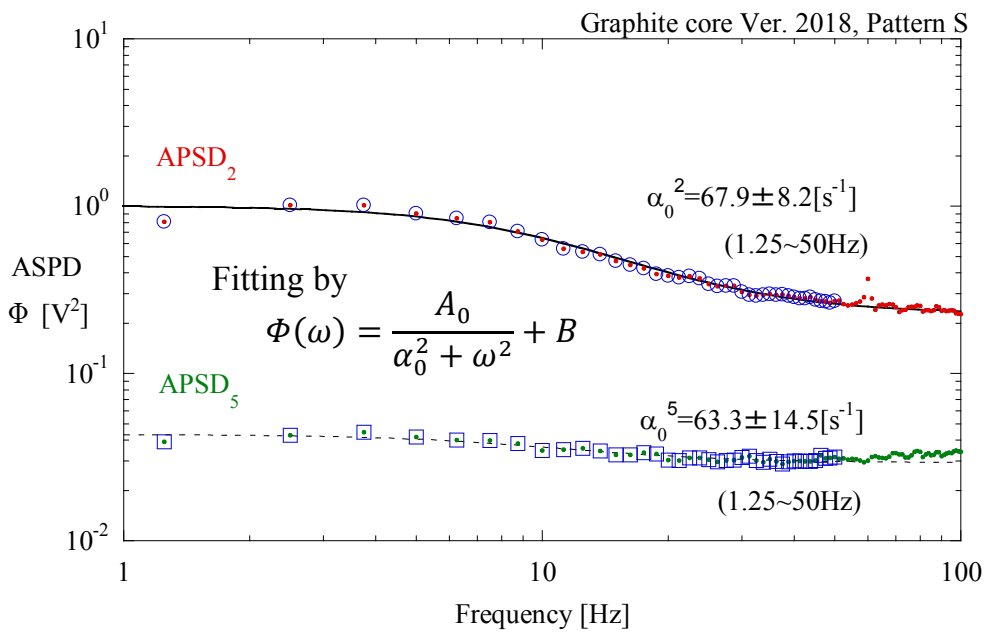

Figure 3. Auto-power spectral density from B2 and B5 in rod pattern S (critical state).

Figure 4 shows measured cross-power spectral density between neutron detector B2 and B5 in rod pattern $\mathrm{S}$ (critical state). The decay constant determined from the cross-power spectral density between the two counters B2 and B5 in rod pattern S also had a consistent value. The attenuation curve of the firstorder lag system of the cross-power spectral density was clearly confirmed compared to that of the autopower spectral density. These results indicate that the same fission correlation information is observed by detectors B2 and B5. 


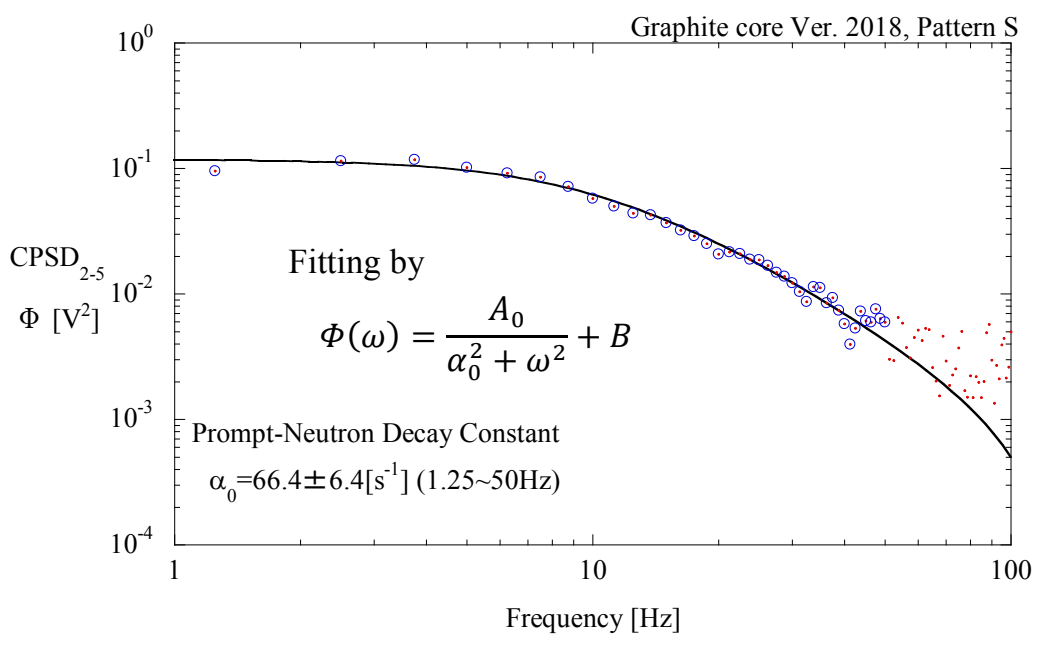

Figure 4. Cross-power spectral density between $B 2$ and $B 5$ in rod pattern S (critical state).

Figure 5 shows measured auto-power spectral densities from neutron detectors B2 and B5 in rod pattern A, and Figure 6 shows measured cross-power spectral density between detectors B2 and B5 in rod pattern A. In rod pattern A, prompt-neutron decay constants were obtained from the auto-power spectral density of detector B2 and the cross-power spectral density between detectors B2 and B5, respectively. The decay constant from auto-power spectral density of detector B5 was not obtained because the fitting error of this case is large. This is due to the fact that the frequency region higher than $100 \mathrm{~Hz}$ of autopower spectral density by the detector B5 has greatly increased due to the direct component (white noise) from the neutron source. The auto-power spectral density by the detector B2 is also increased in the frequency region higher than $200 \mathrm{~Hz}$. It was confirmed that the direct component from the neutron source had reached the position of detector B2, and that the effect of the neutron source in the graphite-reflected core was larger than that of light water- or polyethylene-reflected core.

In rod pattern B, prompt-neutron decay constants were not obtained for all power spectrum densities. The main factor is that neutrons from Am-Be neutron source increased compared to neutrons from the fuel region among the neutrons measured by the detector.

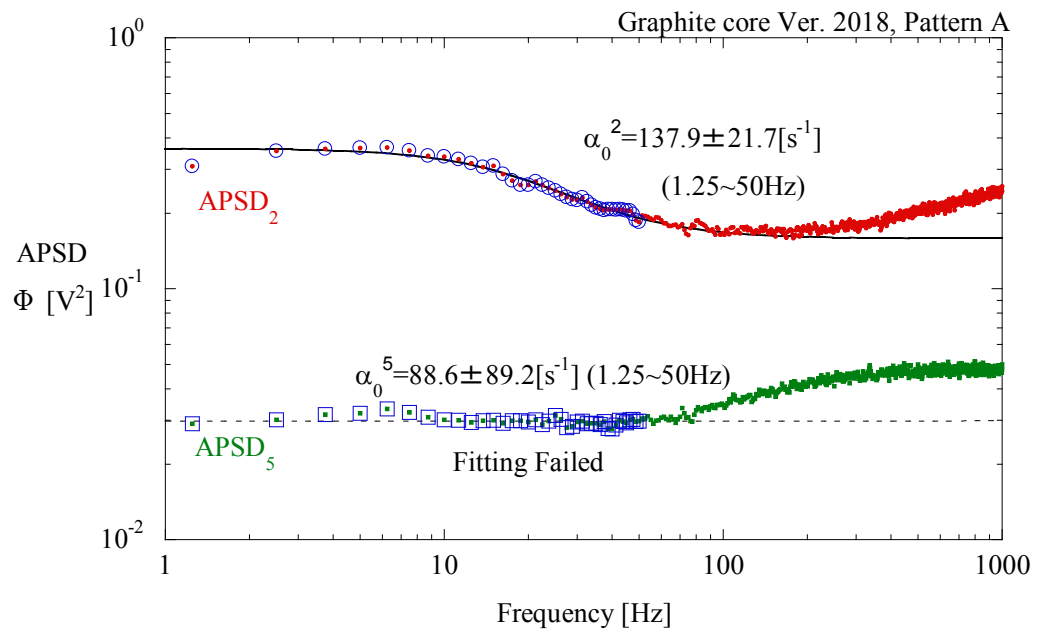

Figure 5. Auto-power spectral density from B2 and B5 in rod pattern A (subcritical state). 


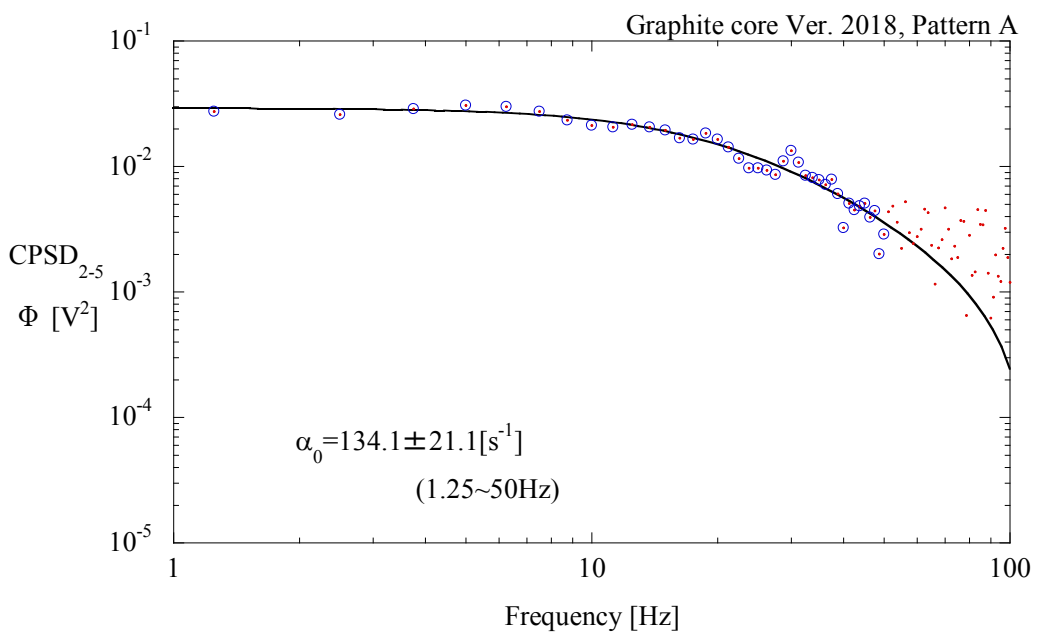

Figure 6. Cross-power spectral density between B2 and B5 in rod pattern A (subcritical state).

Figure 7 shows measured auto-power spectral densities from neutron detectors B4 and B6 in rod pattern C. In rod pattern $\mathrm{C}$, detectors near the fuel region was selected compared to other rod pattern. However, the prompt-neutron decay constants were not obtained from the auto-power spectral densities by detectors B4 and B6. This is because the effect of neutron source is too large as in the case of Pattern B.

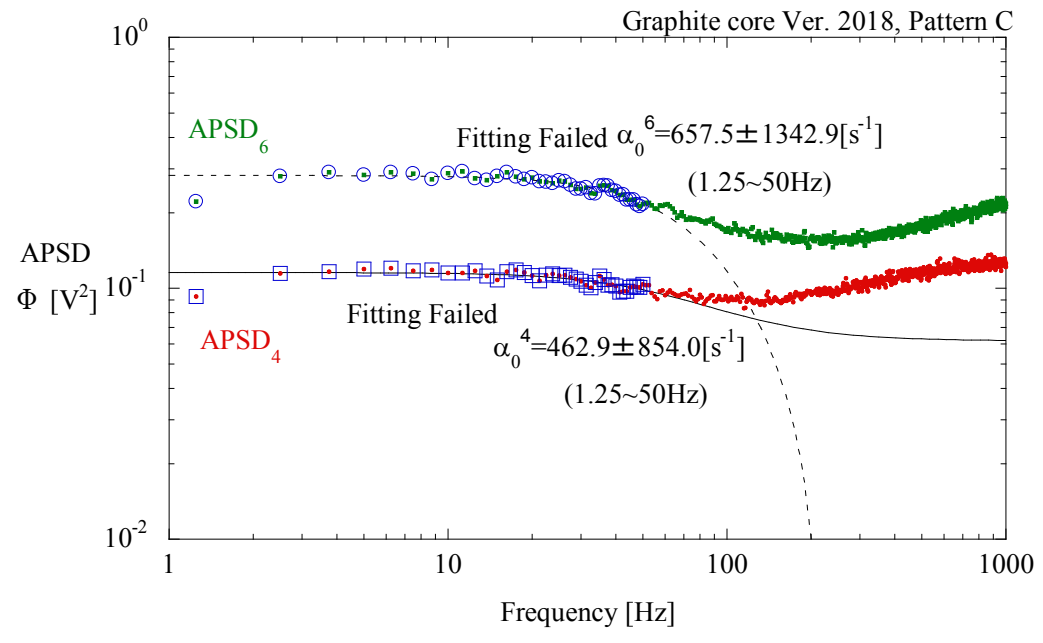

Figure 7. Auto-power spectral density from B4 and B6 in rod pattern C (subcritical state).

Figure 8 shows measured cross-power spectral density between detectors B4 and B6 in rod pattern C. In rod pattern $\mathrm{C}$, prompt-neutron decay constant was obtained from the cross-power spectral density between detectors B4 and B6. As the distance between the two detectors and neutron source is greatly different, the correlation component of the fuel region appears in the cross power spectral density. 


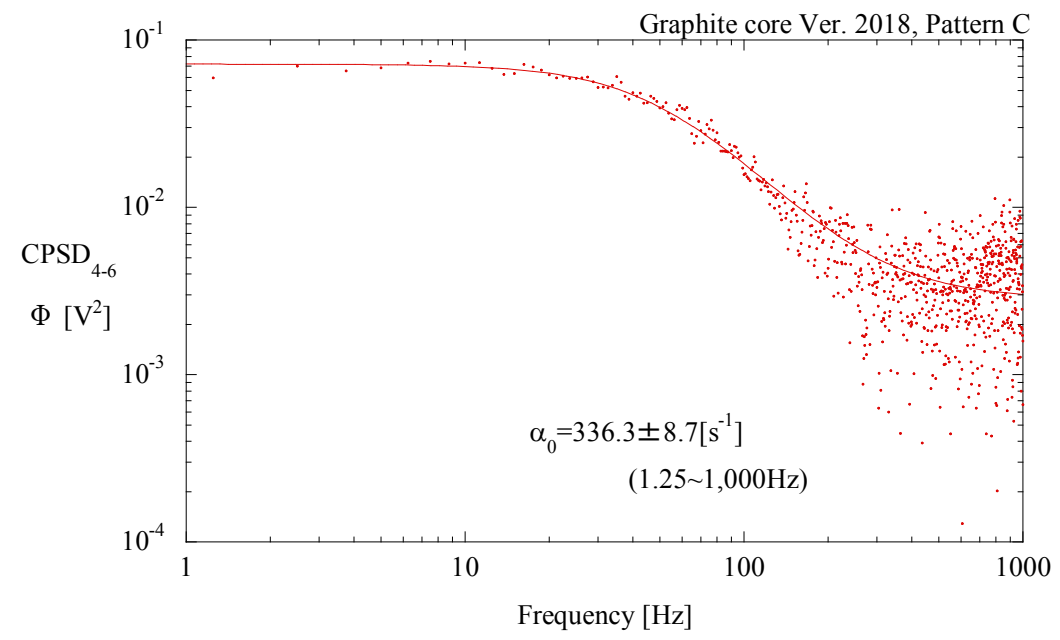

Figure 8. Cross-power spectral density between B4 and B6 in rod pattern C (subcritical state).

The prompt-neutron decay constants inferred from the correlated reactor noise components of the cross and auto-power spectral density are summarized in Table II.

Table II. Prompt-neutron decay constant $\alpha_{0}$.

\begin{tabular}{|c|c|c|c|c|c|}
\hline \multirow{2}{*}{ Rod Pattern } & \multirow{2}{*}{ Detector } & \multicolumn{3}{|c|}{ Prompt- neutron decay constant $\alpha_{0}$} & \multirow{2}{*}{$\begin{array}{c}\text { Reactivity } \\
{[\% \Delta \mathrm{k} / \mathrm{k}]}\end{array}$} \\
\cline { 3 - 5 } & & $\begin{array}{c}\text { ASPD } \\
(\mathrm{B} 2 \text { od B4) }\end{array}$ & $\begin{array}{c}\text { ASPD } \\
(\mathrm{B} 5 \text { or B6) }\end{array}$ & CSPD & \\
\hline S & B2 and B5 & $67.9 \pm 8.2$ & $63.3 \pm 14.5$ & $66.4 \pm 6.4$ & 0 (critical) \\
\hline A & B2 and B5 & $137.9 \pm 21.7$ & Failed & $134.1 \pm 21.1$ & 0.738 \\
\hline B & B2 and B5 & Failed & Failed & Failed & 1.871 \\
\hline C & B4 and B6 & Failed & Failed & $336.3 \pm 8.7$ & 3.942 \\
\hline
\end{tabular}

\section{CONCLUSION}

In order to reactor noise analysis in a light-water or polyethylene reflected core, it was necessary to place a detector close to the fuel region. The graphite reflected core is expected to be able to measure the correlation component of reactor noise with a detector more than $30 \mathrm{~cm}$ away from the fuel region. This is because the mean free path of neutrons in graphite is longer than that of light-water or polyethylene. The purpose of this study was to experimentally confirm the superiority of the detector arrangement in the reactor noise analysis in the graphite-reflected core and the amount of effect by the neutron source. To achieve these goals, a series of reactor noise experiments by power spectrum method were performed on the graphite-reflected core of the KUCA. As a result, the prompt-neutron decay constants were experimentally obtained by using power spectrum analysis with a detector $35 \mathrm{~cm}$ away from the fuel region. The decay constants obtained from power spectrum analysis agrees with each other within fitting error range. However, in the subcritical state, it was confirmed that the effect of neutron source was very large.

\section{ACKNOWLEDGMENTS}

The present work was performed as a joint research program of the KUCA at the Institute for Integrated Radiation and Nuclear Science, Kyoto University. 


\section{REFERENCES}

1. S. Saito, T. Tanaka, Y. Sudo, Design of high temperature engineering test reactor (HTTR). No. JAERI--1332. Japan Atomic Energy Research Inst., 1994.

2. K. Sawa, and S. Ueta, "Research and development on HTGR fuel in the HTTR project." Nuclear Engineering and Design 233.1-3 (2004): 163-172.

3. N. Fujimoto, et al. First criticality prediction of the HTTR by $1 / M$ interposition method. No. JAERICONF--99-006. 1999.

4. N. Fujimoto, et al. Control rod position and temperature coefficients in HTTR power-rise tests. Interim report. No. JAERI-TECH--2000-091. Japan Atomic Energy Research Inst., 2001.

5. Y. Fukaya, M. Goto, S. Nakagawa, et al., "Reactor Physics Experiment in a Graphite-Moderation System for HTGR," Proc of PHYSOR 2020, Cambridge, U.K., March 29 - April 2, (2020).

[to be published]

6. K. Takahashi, K. Nakajima, A. Sakon, et al., "R\&D to improve accuracy of nuclear prediction for HTGR, (2) Inverse Kinetics Analysis for HTTR Simulated Core in KUCA," fall meeting of AESJ, Toyama, Japan, Sept. 11-13, 2J15,(2019).

7. M. M. R. Williams, Random Processes in Nuclear Reactors, (Pergamon Press, Oxford,1974), section 3.6.

8. Y. Nagaya, K. Okumura, T. Sakurai and T. Mori, MVP/GMVP Version 3 : General Purpose Monte Carlo Codes for Neutron and Photon Transport Calculations Based on Continuous Energy and Multigroup Methods, Tokai-mura: Japan Atomic Energy Agency; 2017, (JAEA-Data/Code 2016018).

9. K. Shibata, O. Iwamoto, T. Nakagawa, et al., "JENDL-4.0; A New library for nuclear science and engineering,” J. Nucl. Sci. Techonol., 48(1), pp.1-30 (2011). 